\title{
Searches for supersymmetry in resonance production, R-parity violating signatures and events with long-lived particles with the ATLAS detector
}

\author{
Matthew KING*( Kobe University (JP)) \\ E-mail: kingmglestu.kobe-u.ac.jp
}

\begin{abstract}
Extensions to supersymmetry (SUSY) or the admission of R-parity violation (RPV) introduce new signatures that can be analysed at the LHC. Strongly interacting resonances may decay to jets, sleptons may decay via lepton number violating processes and long-lived particles may be produced. Recent searches for such signatures at ATLAS are presented here. No evidence of any deviation with respect to the Standard Model expectations has been observed, and limits on the respective models have been set. Two results set limits on the RPV models with searches for leptonic signatures in $21 \mathrm{fb}^{-1}$ of $8 \mathrm{TeV}$ data. Three analyses set limits on RPV and extended SUSY models with leptonic and hadronic resonance searches in $\sim 5 \mathrm{fb}^{-1}$ of $7 \mathrm{TeV}$ data. Finally, four searches are presented for signatures of long-lived particles ranging from displaced vertices to stable massive particles in $\sim 5 \mathrm{fb}^{-1}$ of $7 \mathrm{TeV}$ data.
\end{abstract}

XXI International Workshop on Deep-Inelastic Scattering and Related Subjects 22-26 April, 2013

Marseilles, France

\footnotetext{
* Speaker.
} 


\section{Introduction}

Extended Supersymmetry (SUSY) models or the admission of R-parity violation (RPV) can lead to unusual signatures at ATLAS. These may include:

- signatures with a large number of leptons or jets;

- lepton or baryon number violating decays;

- heavy resonances production, leptonic or hadronic in nature;

- long-lived particle production.

ATLAS has recently obtained several results on searches for SUSY, with RPV interpretations, heavy resonances and long-lived particles. Here, an overview is given of the search methods and the current limits on models. All limits quoted are at the $95 \%$ confidence level.

\section{The ATLAS detector}

The ATLAS experiment is one of the two general purpose detectors on the LHC. It is designed to be able to reconstruct events from many different physics processes and can be used to investigate a variety of physical theories.

ATLAS can briefly be described as having 4 concentric detector systems. From the innermost system outwards: the inner detector (ID) tracks charged particles near the beam crossing with sufficient accuracy to reconstruct vertices; the electromagnetic calorimeter (EMcal) stops electrons and photons, measuring their energy; the hadronic calorimeter stops strongly interacting standard model particles, measuring their energy; the muon spectrometer (MS) reconstructs charged tracks outside of the calorimeter system. A solenoid surrounding the ID and a toroidal system interspersed with the MS allow track momentum to be measured.

Further information on ATLAS can be found in reference [1].

\section{Searches with R-parity violating signatures}

Despite R-parity being conserved in many SUSY models, RPV terms are permitted in the superpotential, provided that baryonic and leptonic numbers are not simultaneously violated. The introduction of RPV leads to a new detector phenomenology.

High lepton or jet multiplicity may be indicative of RPV events. The lack of a stable lightest SUSY particle (LSP) generally causes the missing transverse energy $\left(E_{\mathrm{T}}^{\text {miss }}\right)$ signature of RPV events to be reduced or absent compared with R-parity conserving (RPC) events.

Two recent results by ATLAS [2,3] search for high lepton and jet multiplicity final states, analysing $21 \mathrm{fb}^{-1}$ of $8 \mathrm{TeV}$ data. They have low Standard Model (SM) background and can be interpreted in terms of RPV models.

Ref[2] searches for a 4 lepton signature, with a typical decay process as shown in fig. 1a. There are two main signal regions used to set limits on models with different decay couplings; SR0 requires $n_{e, \mu} \geq 4$ and SR1 requires $n_{e, \mu}=3$ and $n_{\tau} \geq 1$. Ref[3] searches for the 2 same-sign lepton signature that may arise from the process shown in fig. $1 \mathrm{~b}$. The leptonic requirement is coupled 


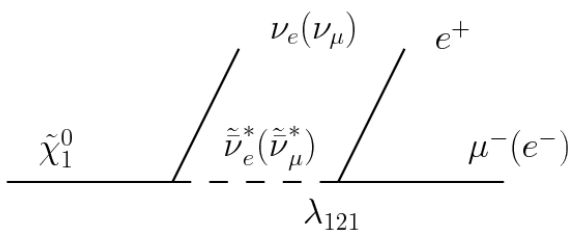

(a) RPV 4 lepton decay [2]

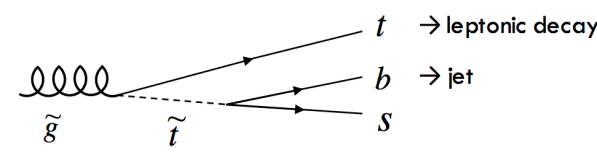

(b) RPV same-sign lepton decay [3]

Figure 1: RPV decay diagrams

with a requirement of $\geq 3 b$-jets and $\geq 4$ jets total. Both analyses also apply cuts on the $E_{\mathrm{T}}^{\text {miss }}$ and transverse invariant mass of events in their signal regions.

No excess beyond the SM expectations is observed.

In ref[2], the result is interpreted in a $\tilde{\chi}^{+} \tilde{\chi}^{-}$"Wino" model and a $\tilde{g} \tilde{g}$ "Gluino" model. When $\lambda_{121} \neq 0$, exclusions of $m_{\tilde{\chi}^{ \pm}} \leq 0.75 \mathrm{TeV}$ and $m_{\tilde{g}} \leq 1.4 \mathrm{TeV}$ were obtained using SR0. When $\lambda_{133} \neq 0$, exclusions of $m_{\tilde{\chi}^{ \pm}} \leq 0.4 \mathrm{TeV}$ and $m_{\tilde{g}} \leq 1.0 \mathrm{TeV}$ were obtained using a combination of signal regions and SR1 respectively. In ref[3] the RPV interpretation of the result excludes $m_{\tilde{g}}<\sim 0.86 \mathrm{TeV}$.

\section{Searches for heavy resonances}

Another possible signature of RPV is the production of unusual resonances. Resonances with final states resulting from lepton number or baryon number violation are strong indications of RPV. Other resonances can be interpreted in terms of RPV or extended SUSY models.

There are three analyses $[4,5,6]$ by ATLAS searching for such resonances in $\sim 5 \mathrm{fb}^{-1}$ of $7 \mathrm{TeV}$ data.

Ref[4] searches for resonances of different generation leptons. Ref[5] searches for pairs of matching 2-jet resonances by finding all potential resonances in events and cutting on their mass difference and angular separation. Ref[6] searches for pairs of resolved or boosted 3-jet resonances. High-mass resonances are expected to produce resolved jets, that may be identified individually, whilst low-mass resonances could lead to a compound jet, that can be analysed by looking for a 3-jet substructure.

In all the cases, no excess beyond the SM expectation is found.

Ref[4] interprets its result in terms of a $\tilde{v}_{\tau}$ decay, leading to limits on combinations of the production and decay couplings $\left(\lambda_{133}^{\prime}, \lambda_{i 3 k}\right)$ and $m_{\tilde{v}_{\tau}}$. Ref[5] sets limits on the production of scalar gluons $\left(g_{s}\right)$. These particles are foreseen to decay to a $g g$ final state in some extended SUSY models, and are excluded in the mass range $150 \mathrm{GeV}<\mathrm{m}_{\mathrm{g}_{\mathrm{s}}}<287 \mathrm{GeV}$. Ref[6] excludes $m_{\tilde{g}}<666 \mathrm{GeV}$ with the analysis of resolved jets and $m_{\tilde{g}}<255 \mathrm{GeV}$ with analysis of boosted jets.

\section{Searches for long-lived particles}

SUSY particles with lifetimes long enough to have a measurable decay length, that may then be detected, are predicted in some models. In RPC models the LSP is stable but undetectable. If the decay of the next to lightest SUSY particle is suppressed, either through mass degeneracy with 
the LSP or through very weak coupling, then its decay length can become measurable. In RPV models the LSP is unstable and may have a measurable decay length.

There are four recent searches for long-lived particles at ATLAS using between $4.4 \mathrm{fb}^{-1}$ and $4.8 \mathrm{fb}^{-1}$ of $7 \mathrm{TeV}$ ATLAS data. The signature of a long-lived particle depends on the scale of the decay length, which varies significantly between the searches.

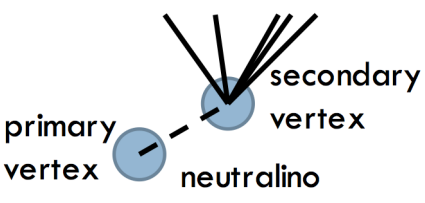

(a) $c \tau \approx 10 \mathrm{~mm}$, displaced vertex

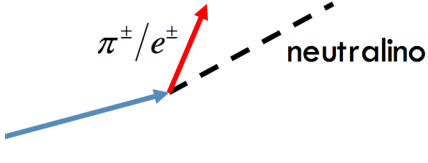

(b) $c \tau \approx 100 \mathrm{~mm}$, disappearing track

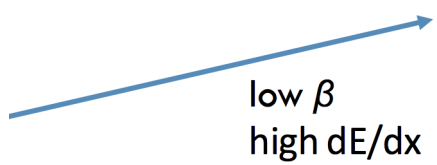

(c) $c \tau>1 \mathrm{~m}$, stable massive particle

Figure 2: Typical signatures of long-lived particles with different decay lengths $(c \tau)$.

Ref[7] searches for $\tilde{\chi}^{0}$ decays to $\mu+$ jets at a displaced vertex (fig. 2a). $\tilde{\chi}^{0}$ decays are identified by searching for reconstructed vertices with transverse displacement $d_{0}>1.5 \mathrm{~mm}$ from the interaction point. Ref[8] also searches for displaced vertices, but for decays to a photon and a gravitino. The gravitino is undetectable, so the vertex cannot be reconstructed. Instead, photons with high transverse energy not pointing back towards the interaction point (non-pointing) are searched for. Additionally, evidence of late photon arrival is searched for as a cross check against the nonpointing result. This analysis is only feasible at ATLAS due to the high EMcal pointing resolution the ATLAS EMcal design affords. Ref[9] searches for $\tilde{\chi}^{ \pm}$decays into a neutralino and soft pion, experimentally being detectable as disappearing tracks (fig. $2 b$ ). Such particles would decay inside the ID and are identified by finding tracks well reconstructed in the inner ID layers, but with low numbers of hits in the outer ID layers. Ref[10] searches for particles that travel completely through ATLAS before decaying (fig. 2c). Such particles can be identified by large $\frac{\mathrm{d} E}{\mathrm{~d} x}$ deposition in the ID and calorimeter and low measured velocity in the calorimeter and MS. Long-lived stau $(\tilde{\tau})$ in gauge mediated SUSY breaking (GMSB) models are searched for using velocity measurements alone. Long-lived R-hadrons in more general SUSY are searched for using combinations of velocity and $\frac{d E}{d x}$ measurements and may have shorter lifetimes.

No excess beyond the SM expectation is found.

Ref[7] leads to an exclusion on squark masses below $\sim 700 \mathrm{GeV}$ for $\tilde{\chi}^{0}$ decay lengths between $1 \mathrm{~mm}$ and $1000 \mathrm{~mm}$. Ref[8] excludes $m_{\tilde{\chi}^{0}}<\sim 230 \mathrm{GeV}$ for $0.4<\tau_{\tilde{\chi}^{0}}<2 \mathrm{~ns}$. No evidence of late photon detection was found. Ref[9] excludes $m_{\tilde{\chi}^{0}}<\sim 100 \mathrm{GeV}$ at the most probable decay time of $\tau \approx 0.3 \mathrm{~ns}$. The most stringent limit, excluding $m_{\tilde{\chi}^{0}}<\sim 260 \mathrm{GeV}$, can be set for decay times of $\tau \approx 3$.0ns. Ref[10] excludes long-lived GMSB $\tilde{\tau}$ with $m_{\tilde{\tau}}<\sim 300 \mathrm{GeV}$ for $\tan \beta=5$ - 20. Gluino masses below $985 \mathrm{GeV}$ are excluded by the full detector R-hadron search, as are stop (sbottom) masses below $683 \mathrm{GeV}(612 \mathrm{GeV})$. Searches over a smaller detector distance account for Rhadrons changing charge during flight or interacting with the detector and set similar limits on $m_{\tilde{g}}$, $m_{\tilde{t}}$ and $m_{\tilde{b}}$.

\section{Summary}

Certain SUSY models predict interesting signatures that are being searched for at ATLAS. 
These signatures are varied in nature and can require some unusual and challenging techniques to be identified.

No evidence for SUSY signatures has been found in resonance production, RPV and longlived particle searches. Updated results on the 2012 data are awaited soon.

\section{References}

[1] The ATLAS Collaboration, The ATLAS Experiment at the CERN Large Hadron Collider, JINST 3 S08003 (2008)

[2] The ATLAS Collaboration, Search for supersymmetry in events with four or more leptons in $21 \mathrm{fb}^{-1}$ of pp collisions at $\sqrt{s}=8 \mathrm{TeV}$ with the ATLAS detector, ATLAS-CONF-2013-036 (2013)

[3] The ATLAS Collaboration, Search for strongly produced superpartners in final states with two same sign leptons with the ATLAS detector using $21 \mathrm{fb}^{-1}$ of proton-proton collisions at $\sqrt{s}=8 \mathrm{TeV}$, ATLAS-CONF-2013-007 (2013)

[4] The ATLAS Collaboration, Search for a heavy narrow resonance decaying to e $\mu$, e $\tau$, or $\mu \tau$ with the ATLAS detector in pp collisions at the LHC, PLB 723, 15 (2013), [arXiv:1212.1272]

[5] The ATLAS Collaboration, Search for pair-produced massive coloured scalars in four-jet final states with the ATLAS detector in proton-proton collisions at $\sqrt{s}=7 \mathrm{TeV}$, EPJC 73, 2263 (2013), [arXiv:1210.4826]

[6] The ATLAS Collaboration, Search for pair production of massive particles decaying into three quarks with the ATLAS detector in $\sqrt{s}=7 \mathrm{TeV}$ pp collisions at the LHC, JHEP 12, 086 (2013), [arXiv:1210.4813]

[7] The ATLAS Collaboration, Search for long-lived, heavy particles in final states with a muon and multi-track displaced vertex in proton-proton collisions at $\sqrt{s}=7 \mathrm{TeV}$ with the ATLAS detector, PLB 719, 280 (2013), [arXiv:1210.7451]

[8] The ATLAS Collaboration, Search for non-pointing photons in the diphoton and missing transverse energy final state in $7 \mathrm{TeV}$ proton-proton collisions using the ATLAS detector, CERN-PH-EP-2013-049 (2013), accepted by PRD, arXiv:1304.6310 (2013)

[9] The ATLAS Collaboration, Search for direct chargino production in anomaly-mediated supersymmetry breaking models based on a disappearing-track signature in pp collisions at $\sqrt{s}=7 \mathrm{TeV}$ with the ATLAS detector, JHEP 01, 131 (2013), [arXiv:1210.2852]

[10] The ATLAS Collaboration, Searches for heavy long-lived sleptons and R-Hadrons with the ATLAS detector in pp collisions at $\sqrt{s}=7 \mathrm{TeV}$, PLB 720, 277 (2013), [arXiv:1211.1597] 